\title{
Ectopic Expression of Gs5PTase8, a Soybean Inositol Polyphosphate 5-Phosphatase, Enhances Salt Tolerance in Plants
}

\author{
Qi Jia ${ }^{1,2, *}$, Song Sun ${ }^{1}$, Defeng Kong ${ }^{1}$, Junliang Song ${ }^{1}$, Lumei $\mathrm{Wu}^{1}{ }^{1}$, Zhen Yan ${ }^{1}$, Lin Zuo ${ }^{1}$, \\ Yingjie Yang ${ }^{1}$, Kangjing Liang ${ }^{1}$, Wenxiong Lin ${ }^{1,2}$ and Jinwen Huang ${ }^{1, *}$ \\ 1 Key Laboratory for Genetics Breeding and Multiple Utilization of Crops, Ministry of Education/College of \\ Agriculture, Fujian Agriculture and Forestry University, Fuzhou 350002, China; sunsong07@163.com (S.S.); \\ kongyuanyuan00210@163.com (D.K.); xgrz@163.com (J.S.); Wu_lumei@163.com (L.W.); \\ zhenyan252858@163.com (Z.Y.); 17628439383@163.com (L.Z.); y2908363303@126.com (Y.Y.); \\ liangkj_2005@126.com (K.L.); lwx@fafu.edu.cn (W.L.) \\ 2 Key Laboratory of Crop Ecology and Molecular Physiology (Fujian Agriculture and Forestry University), \\ Fujian Province University, Fuzhou 350002, China \\ * Correspondence: jiaqi@fafu.edu.cn (Q.J.); hqliuyh@163.com (J.H.)
}

Received: 27 December 2019; Accepted: 1 February 2020; Published: 4 February 2020

\begin{abstract}
Inositol polyphosphate 5-phosphatases (5PTases) function in inositol signaling by regulating the catabolism of phosphoinositol derivatives. Previous reports showed that 5PTases play a critical role in plant development and stress responses. In this study, we identified a novel 5PTase gene, Gs5PTase8, from the salt-tolerance locus of chromosome 3 in wild soybean (Glycine soja). Gs5PTase8 is highly up-regulated under salt treatment. It is localized in the nucleus and plasma membrane with a strong signal in the apoplast. Ectopic expression of Gs5PTase8 significantly increased salt tolerance in transgenic BY-2 cells, soybean hairy roots and Arabidopsis, suggesting Gs5PTase8 could increase salt tolerance in plants. The overexpression of Gs5PTase8 significantly enhanced the activities of catalase and ascorbate peroxidase under salt stress. The seeds of Gs5PTase8-transgenic Arabidopsis germinated earlier than the wild type under abscisic acid treatment, indicating Gs5PTase8 would alter ABA sensitivity. Besides, transcriptional analyses showed that the stress-responsive genes, AtRD22, AtRD29A and AtRD29B, were induced with a higher level in the Gs5PTase8-transgenic Arabidopsis plants than in the wild type under salt stress. These results reveal that Gs5PTase8 play a positive role in salt tolerance and might be a candidate gene for improving soybean adaptation to salt stress.
\end{abstract}

Keywords: inositol polyphosphate 5-phosphatase; soybean; salt tolerance; abscisic acid; BY-2; Arabidopsis; hairy roots

\section{Introduction}

Cultivated soybean (Glycine max) is an important economic crop as a main source of dietary protein and oil. However, its development and agricultural productivity could be severely restricted by salt stress [1,2]. Unfortunately, around 20\% irrigated land is salt affected and the salt-affected regions continue to expand $[3,4]$. Thus, it is necessary to improve salt tolerance in cultivated soybean. On the other hand, wild soybean (Glycine soja), the close related species of Glycine max, could confer higher tolerance to salinity or other stresses. Wild soybean could supply resistant gene resources for molecular breeding in soybean. It is meaningful to identify new salt-tolerance genes from Glycine soja and characterize their molecular mechanism.

Inositol polyphosphate 5-phosphatases (5PTases) are signal modifying enzymes with the conserved inositol polyphosphate phosphatase catalytic (IPPc) domain, which could hydrolyze the phosphate 
bond at the 5 position of the inositol ring from inositol phosphate or phosphoinositide $[5,6]$. Thus, the 5PTases have been suggested to inhibit or terminate inositol signaling, correspondingly altering abscisic acid (ABA) signaling, $\mathrm{Ca}^{2+}$ oscillation, and redox homeostasis [7-9]. Previous reports have illustrated that 5PTases participate in various processes of development and stress responses in plants $[7,10-12]$.

The 5PTase genes are widespread among plants. For example, there are 15 members in Arabidopsis thaliana, 21 in rice and 39 in soybean [13]. Until now, the knowledge on the functions of plant 5PTases is still limited, and most is from the model plant Arabidopsis by examining the plants containing gain or loss of function in a certain 5PTase. At5PTase7 and At5PTase9 have been identified to take a crucial role in salt tolerance [14,15]. Mutations in At5PTase7 or AtPTase9 would result in increased salt sensitivity, whereas overexpression of At5PTase 7 or AtPTase9 would enhance salt tolerance in Arabidopsis [14,15]. Both of the At5ptase7 and Atptase9 mutants would disturb reactive oxygen species (ROS) production and the expression of salt-responsive genes. Besides, the Atptase9 mutants also showed reduced $\mathrm{Ca}^{2+}$ influx and decreased fluid-phase endocytosis, suggesting At5PTase7 and AtPTase9 have non-redundant roles in the regulation of plant salt tolerance $[14,15]$.

On the other hand, GmSAL1, a soybean inositol 1-phosphatases, has been found to be involved in salt and drought response by the hydrolysis of inositol-1,4,5-trisphosphate ( $\operatorname{Ins}_{3}$ ) [16], suggesting the inositol signaling would also play a role in stress responses in soybean. The components including 5PTases, involved in inositol signaling, would be good candidates for soybean breeding of salt tolerance as well. However, the function of most 5PTases remains unknown in soybean.

Recently, a salt-tolerance locus has been identified in chromosome 3 of Glycine soja and the salt-tolerant Glycine max varieties [17-19]. In order to dig out more salt-tolerance genes, we performed post-genomonic analyses on this locus. In this study, a novel soybean 5PTase gene, Gs5PTase8, has been identified from this salt tolerance locus due to its high induction by salinity in the Glycine soja accession W05. The analyses of sequences showed that Gs5PTase8 contains the two conserved domain as the other known 5PTases, indicating it is a potential 5PTase. Its effects on plant salt responses were monitored via ectopic expression in BY-2 cells, soybean hairy roots, and Arabidopsis thaliana. Its impacts on the activities of antioxidant enzymes, ABA signaling, and stress-responsive gene expression were also determined here.

\section{Results}

\subsection{Gs5PTase8 Encoding a Putative Inositol Polyphosphate 5-Phosphatas is Induced by Salt Stress}

A novel salt tolerance locus has been identified in the chromosome 3 of soybean previously [17-19]. From the transcriptome data of soybean under salt stress [20], we identified a salt-induced gene, Gs5PTase8 (XM_028369920), in the salt tolerance locus of the G. soja accession W05. To verify the induction of Gs5PTase8 by salinity, the expression analyses were performed in W05 by qRT-PCR (Figure 1A). The results showed that the expression of Gs5PTase 8 was highly up-regulated, especially at $24 \mathrm{~h}$ after salt treatment in both leaves and roots, suggesting Gs5PTase8 would be involved in salt responses.

Gs5PTase8 was then cloned from W05. The cDNA sequence contains 1482 bp encoding a peptide of 493 aa. The BLASTP searches showed that its closest homolog in Arabidopsis thaliana is At5PTase8, so the gene has been named as Gs5PTase8 (Figure 1B). Its counterpart in cultivated soybean is Glyma.03G173000, named as Gm5TPase8 in this study. The analyses of phylogenic relationship were performed with the full-length amino acid sequences of Gs5PTase8, Gm5TPase8, Arabidopsis 5PTases, and other well-known 5PTases from human (Homo sapiens), rat (Rattus norvegicus), and yeast (Saccharomyces cerevisiae) using the neighbor-joining method with 1000 replications of bootstrap tests by MEGA6.0 (Figure 1B). It revealed that Gs5PTase8 and Gm5PTase8 were closer to the Arabidopsis At5PTases without WD-repeats (WD40 domain, cd00200, the GenBank conserved domain database) than those At5PTases with WD-repeats, and also closer to human Type II 5PTase than the other human 5PTases. The sequences analysis indicated that Gs5PTase8 contained the conserved domain I and 
II of the 5-phoshatase catalytic domains as the other known 5PTases, suggesting Gs5PTase8 encodes a putative inositol polyphosphate 5-phosphatase (Figure 1C).

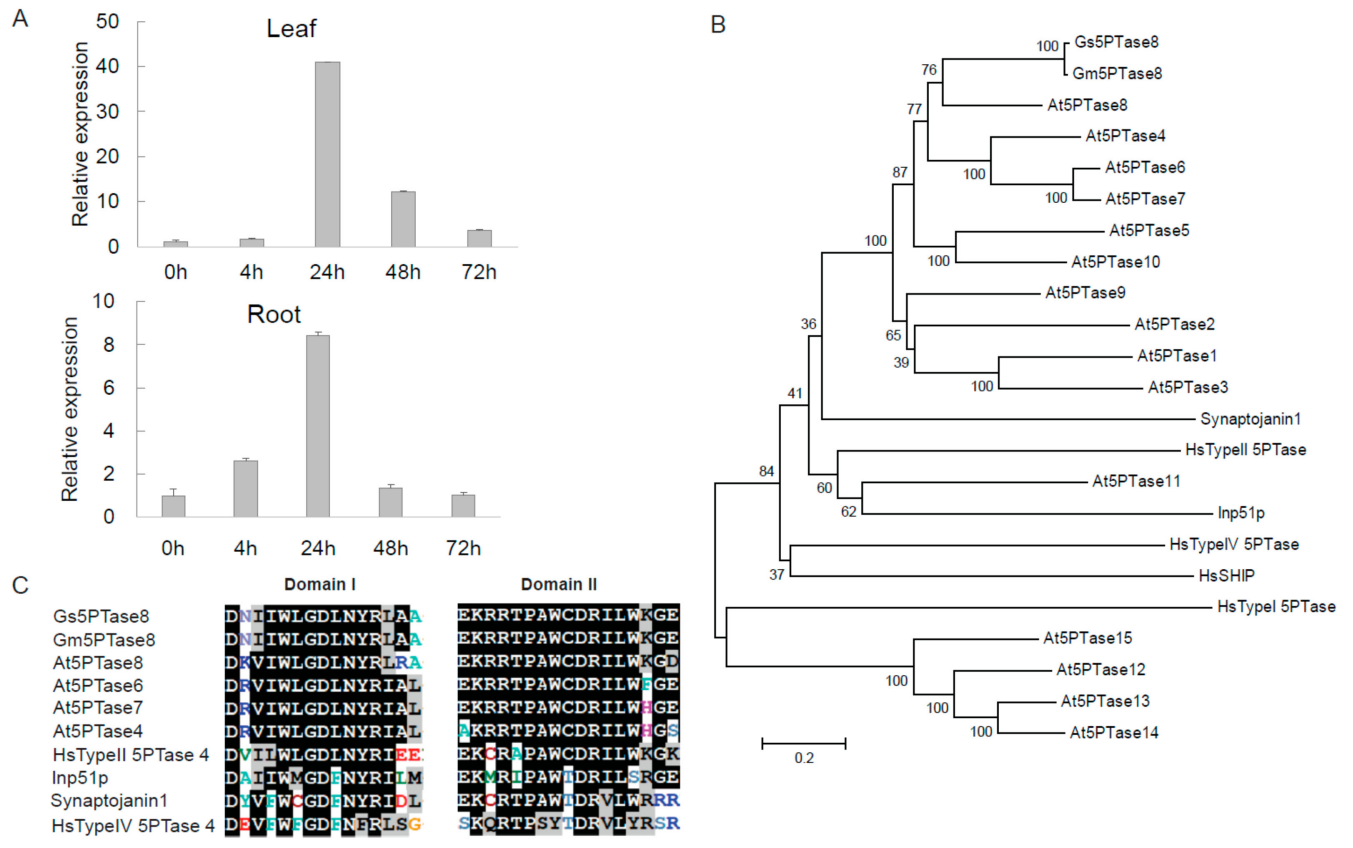

Figure 1. Gs5PTase8, a putative inositol polyphosphate 5-phosphatase, is induced by salt. (A) The expression of Gs5PTase8 in the Glycine soja accession W05 under salt treatment. Leaves and roots were collected separately after the salt treatments of $0.9 \% \mathrm{NaCl}(w / v)$ at $0 \mathrm{~h}, 4 \mathrm{~h}, 24 \mathrm{~h}, 48 \mathrm{~h}$, and $72 \mathrm{~h}$. The data were normalized to the soybean reference gene ELF1b. The value of the samples collected at $0 \mathrm{~h}$ was set at 1. (B) Phylogenic tree of Gs5PTase8 and its homologs using neighbor-joining method with 1000 replications of bootstrap tests by MEGA6.0. The scale bar indicates 0.2 amino acid substitutions per residue. (C) Sequence alignments of the two conserved 5-phoshatase catalytic domains of Gs5PTase8 and its homologs. The conserved amino acid residues are shaded in black and the similar residues in gray. Gs5PTase8 is the protein from Glycine soja (accession W05), Gm5PTase8 from Glycine max (accession Williams 82 / C08), and At5PTases from Arabidopsis thaliana.Gs5PTase8: XM_028369920; Gm5PTase8: Glyma.03G173000; At5PTase1: At1G34120; At5PTase2: At4G18010; At5PTase3: At1G71710; At5PTase4: At3G63240; At5PTase5: At5G65090; At5PTase6: At1G05470; At5PTase7: At2G32010; At5PTase8: At2g37440; At5PTase9: At2G01900; At5PTase10: At5g04980; At5PTase11: At1G47510; At5PTase12: At2G43900; At5PTase13: At1G05630; At5PTase14: At2G31830; At5PTase15: At1G65580; HsTypeI 5PTase: X77567 (Hs represents for Homo sapiens); HsTypeII 5PTase: M74161; HsTypeIV 5PTase: AF187891; HsSHIP: U57650; Synaptojanin1: U45479 (from Rattus norvegicus); Inp51p: NP_012264 (from Saccharomyces cerevisiae).

\subsection{Ectopic Expression of Gs5PTase8 Increased Salt Tolerance in Plants}

In order to investigate its function in plant salt responses, the effects of Gs5PTase8 were assessed by the utilization of the three transformation systems of BY-2 cell lines, soybean hairy roots and Arabidopsis thaliana. The functional analysis in salt responses was performed in BY-2 cells containing the transgenes of Gs5PTase8 (Figure 2). The expression of the transgenes was confirmed by qRT-PCR. The dead cells were visualized by trypan blue staining. Under normal growth conditions, all the cell lines have around $98 \%$ survival rates. Under $100 \mathrm{mM} \mathrm{NaCl}$ treatments, all the transgenic lines of Gs5PTase8 exhibited significantly higher survival rates than the wild type (WT) and the vector-only control line $(\mathrm{V})$. 

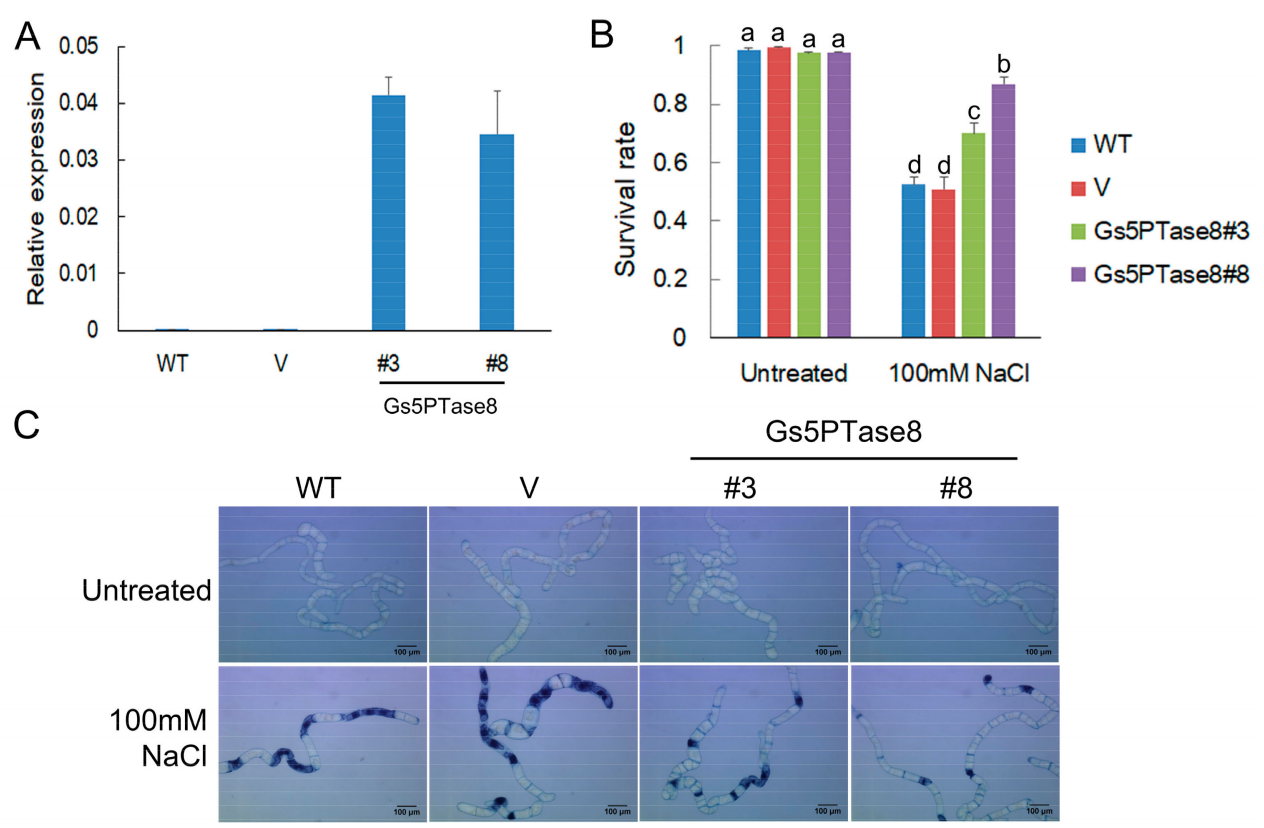

Figure 2. Cell viability assay of BY-2 cells under salt treatment. Four-day-old BY-2 cells cultured in MS medium were used, including the wild type (WT), the vector-only control line (V), two independent Gs5PTase8 transgenic lines (Gs5PTase8\#3 and Gs5PTase8\#8). The cells were treated or untreated with $100 \mathrm{mM} \mathrm{NaCl}$ for $20 \mathrm{~h}$ and the dead cells were visualized by trypan blue staining. (A) The expression of transgenes $\left(2^{-\Delta \mathrm{Ct}}\right)$ were validated by qRT-PCR. The data were normalized to the tobacco reference gene L25. (B) The quantitative analysis of survival rates. For each sample, around 200 300 cells were counted randomly. Three replicates of each experiment were performed. Values are mean \pm SE. The data were analyzed using ANOVA followed by the Duncan's post hoc test $(p<0.05)$. The significant differences were indicated by different letters above the bars. (C) The images of the BY-2 cells by trypan blue staining. Scale bars indicate $100 \mu \mathrm{m}$.

The soybean cotyledons were infected for hairy root induction by the wild type of $A$. rhizogenes stain K599 (CK), K599 containing the empty binary vector pTEV8 (V) or K599 containing the recombinant construct V7-Gs5PTase8 (Gs5PTase8), respectively. The transformation of hairy roots were confirmed by PCR genotyping (Figure 3A). Nine roots were positive transformants in 10 roots, indicating that the transformation was mainly successful. All the hairy roots grew well with no obvious difference under normal growth condition, whereas the growth of Gs5PTase 8 transformed hairy roots was inhibited at a less level with more fresh weight than that of the controls under $150 \mathrm{mM} \mathrm{NaCl}$ (Figure 3). The results revealed that the overexpression of Gs5PTase8 alleviated salt stress in soybean hairy roots. 


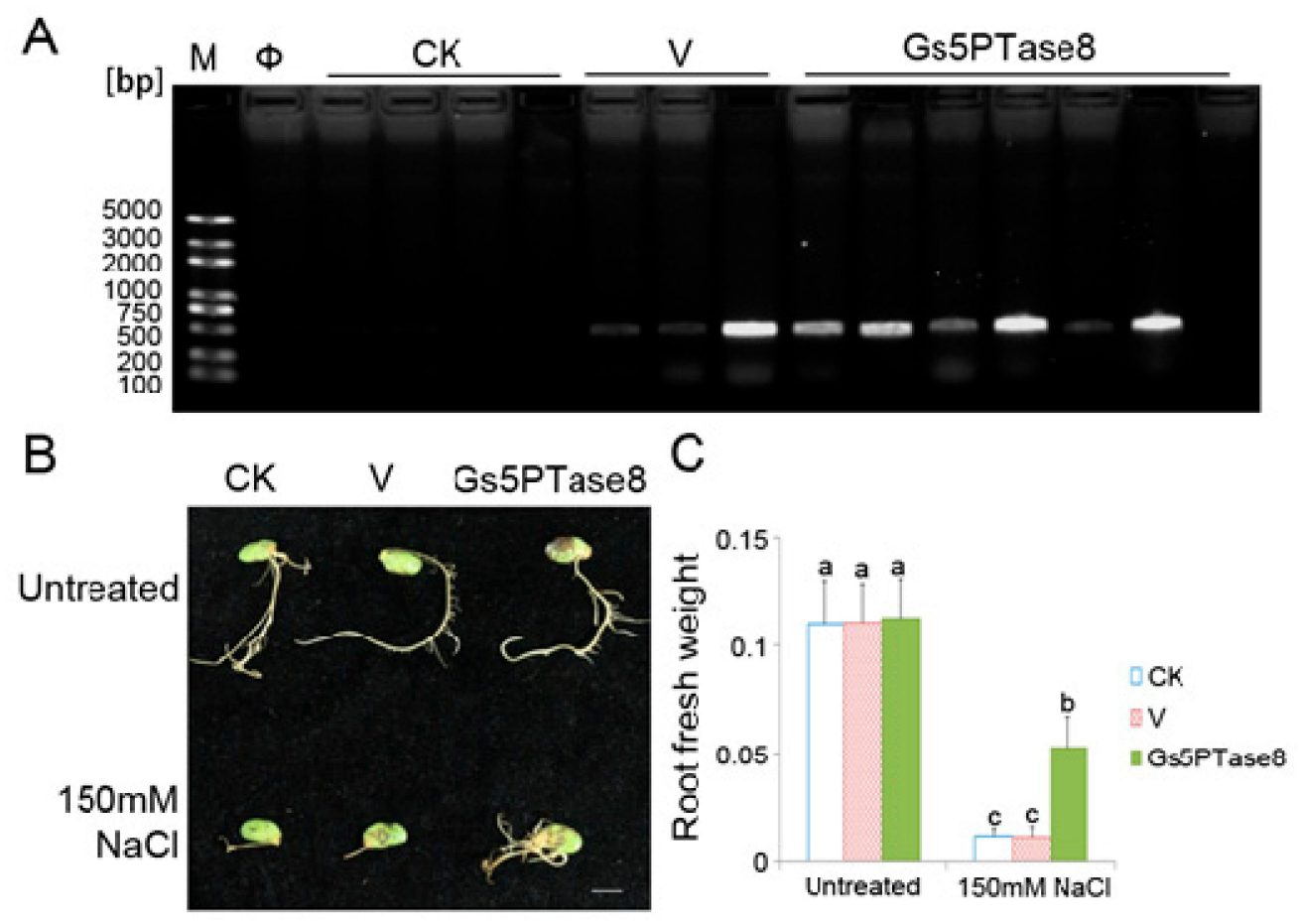

Figure 3. Growth characteristics of soybean hairy roots under salt treatment. The cotyledons of the geminated soybean seeds were infected with the wild type of A. rhizogenes stain K599 (CK), K599 containing the empty vector pTEV8 (V) or K599 containing the recombinant construct V7-Gs5PTase8 (Gs5PTase8), respectively. The cotyledons with the hairy roots of around $1 \mathrm{~cm}$ were selected for salt treatment. The selected hairy roots were grown with $1 / 2$ Hoagland solution containing $0 \mathrm{mM} \mathrm{NaCl}$ and $150 \mathrm{mM} \mathrm{NaCl}$ for 10 days. (A) PCR verification of the positive transformants. The primers of the NPTII gene (kanamycin selection marker) from the binary vector pTEV8 were used for the genotyping PCR. The successful transformants of the empty vector and the recombinant construct would have a PCR product of 400 bps. M represents for DNA marker; $\Phi$, Milli-Q water control of PCR reaction; bp, base pairs. (B) Phenotype of hairy roots. Scale bar indicates $1 \mathrm{~cm}$. (C) Fresh weight of hairy roots. Sample means with standard errors (SE) were calculated with three replicates each containing 5-6 soybean cotyledons. The significant tests were performed using ANOVA (Duncan's test, $p<0.05$ ). The significant differences were indicated by different letters above the bars.

Three independent homozygous transgenic Arabidopsis lines of Gs5PTase8 were obtained for functional analyses under salt stress (Figure 4A). Under normal growth conditions, the seeds of all the lines germinated at a similar rate in 2 days after stratification and there are no significant growth difference between them. Under salt treatment, the germination rates of all the Gs5PTase8-transgenic lines were significantly higher than the rates of the wild type (Col-0), especially at $3.5 \mathrm{~d}$ after stratification (Figure 4B,C). Moreover, when the 3-day seedlings were treated by $175 \mathrm{mM} \mathrm{NaCl}$ for one week, the results showed that the Gs5PTase8-transgenic plants had significantly higher survival rates and relative chlorophyll contents comparing to the wild type (Figure 4D-F). The results demonstrated that the ectopic expression of Gs5PTase8 could increase salt tolerance in Arabidopsis as well, in consisted with its function in BY-2 cells and soybean hairy roots. 


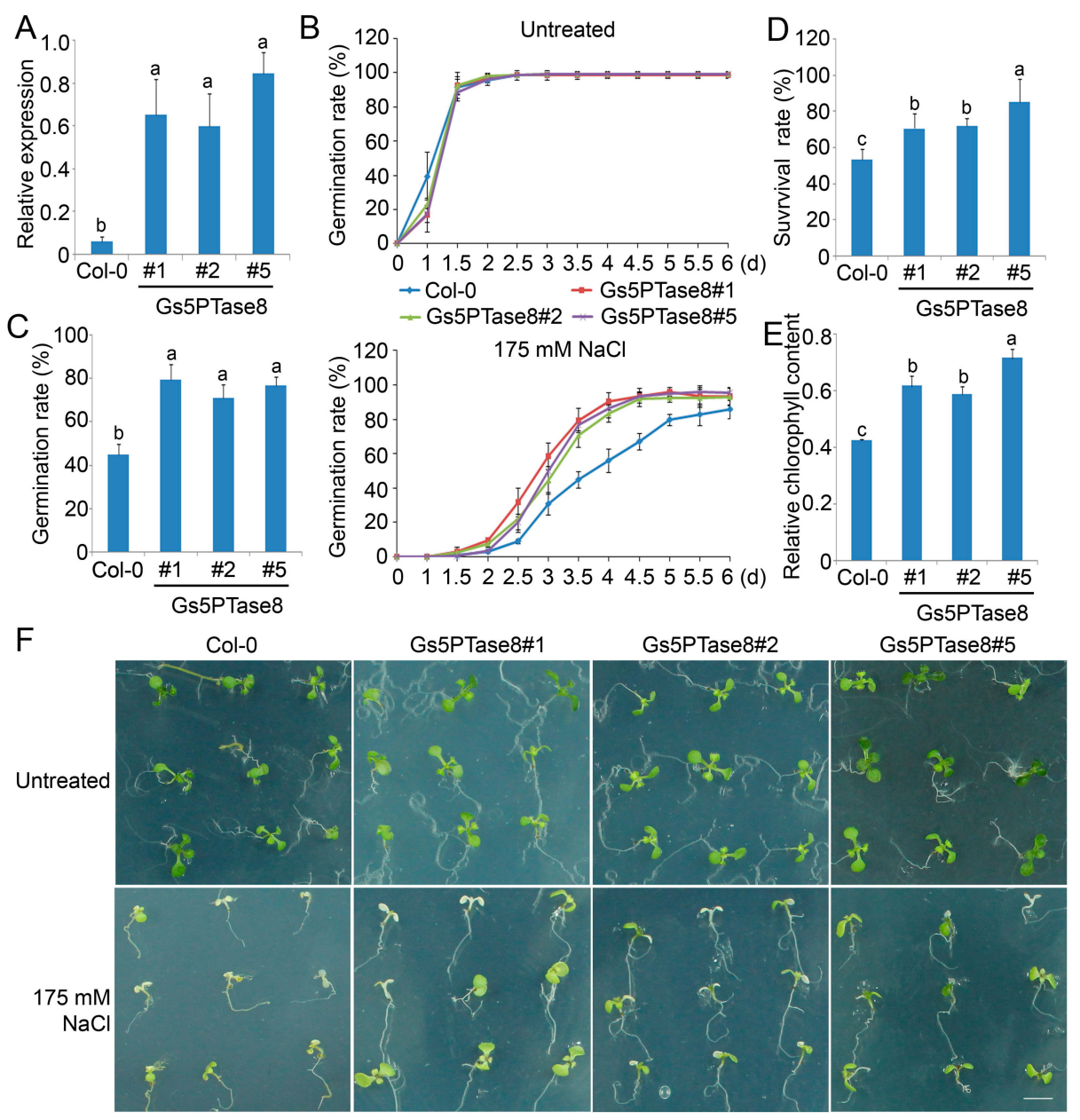

Figure 4. Phenotype of three independent Gs5PTase8-transgenic Arabidopsis lines (\#1, \#2, \#5) under salt treatment. (A) The expression of Gs5PTase8 $\left(2^{-\Delta \mathrm{Ct}}\right)$ were validated in the transgenic lines by qRT-PCR. The data were normalized to the Arabidopsis reference gene ACT2. (B) Arabidopsis seeds were sowed on the $1 / 2 \mathrm{MS}$ agar plates with or without $175 \mathrm{mM} \mathrm{NaCl}$ and were stratified at $4{ }^{\circ} \mathrm{C}$ for $3 \mathrm{~d}$. The germination rates were monitored every $12 \mathrm{~h}$ for 6 days after shift to $21^{\circ} \mathrm{C}$. (C) The statistical analysis of the germination rates were represented at $3.5 \mathrm{~d}$ post stratification. In the germination assays, around 150 seeds were used for each sample. At least three independent experiments were performed. (D) The survival rates after salt treatment. Three-day seedlings were treated by $175 \mathrm{mM} \mathrm{NaCl}$ for one week. Three replicates were performed with 25 plants for each variant. (E) The relative chlorophyll content was shown as the ratio of the chlorophyll content from the untreated plants compared to that from the $175 \mathrm{mM} \mathrm{NaCl}$ treated survival plants for each line. (F) Phenotype of the plants after the survival assays. Scale bar indicates $1 \mathrm{~cm}$. The data were analyzed using ANOVA by the Duncan's post hoc test $(p<0.05)$. The significant differences were indicated by different letters above the bars.

\subsection{Enhanced Activities of Antioxidant Enzymes in Gs5PTase8-Transgenic Arabidopsis}

The evidences suggested that salinity would lead to oxidative stress [3]. To assess the contribution of antioxidant enzymes in reduction of oxidative stress, the activities of catalase (CAT), ascorbate peroxidase (APX), peroxidase (POD), and superoxide dismutase (SOD) were measured in the wild type and the three Gs5PTase8-transgenic Arabidopsis lines. As shown in Figure 5, the application of salt stress increased the four ROS scavenging enzymes more or less. Among them, the activities of CAT and APX were significantly higher in the Gs5PTse8-transgenic lines than the wild type. It demonstrated that the overexpression of Gs5PTse8 could enhance the activities of CAT and APX under salt treatment, probably to alleviate the following oxidative stress caused by salinity. 
A

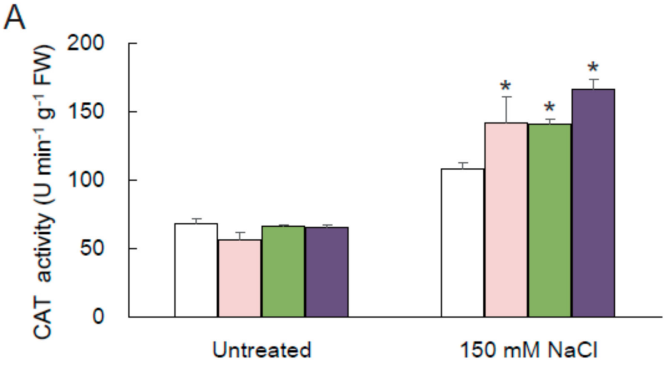

$\square$ Col-0 $\square$ Gs5PTase8\#1 $\square$ Gs5PTase8\#2 $\square$ Gs5PTase8\#5

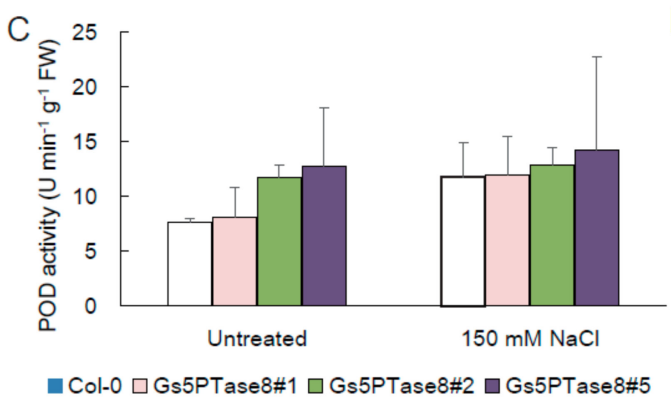

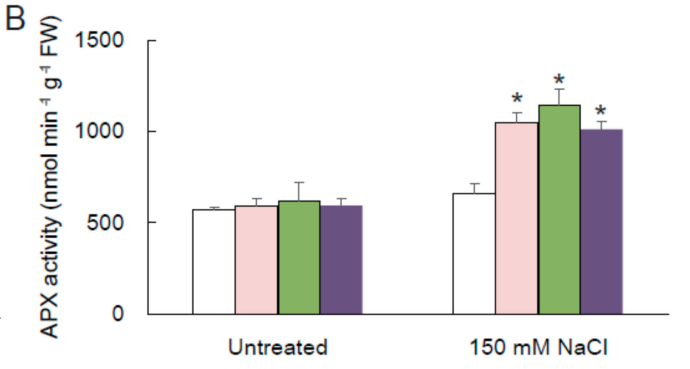

$\square$ Col-0 $\square$ Gs5PTase8\#1 $\square$ Gs5PTase8\#2 $\square$ Gs5PTase8\#5

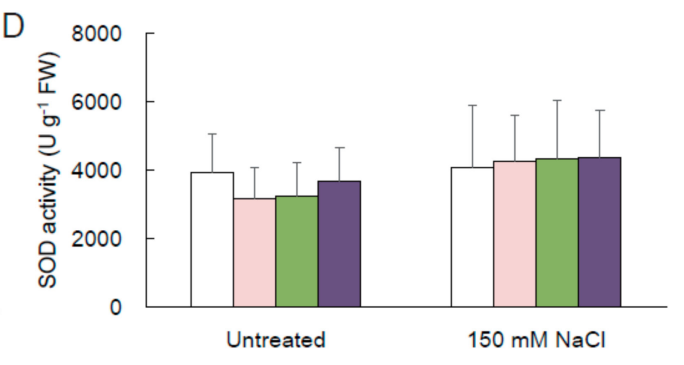

$\square$ Col-0 $\square$ Gs5PTase8\#1 $\square$ Gs5PTase8\#2 $\square$ Gs5PTase8\#5

Figure 5. Activities of antioxidant enzymes in Gs5PTase8-transgenic Arabidopsis under salt treatment. The ten-day-old Arabidopsis seedlings were treated with or without $150 \mathrm{mM} \mathrm{NaCl}$ for $24 \mathrm{~h}$. (A) CAT activity, (B) APX activity, (C) POD activity, and (D) SOD activity. The data were analyzed using ANOVA by the Dunnett's post hoc test. Asterisk indicates a significant difference between the wild type and the transgenic lines $(p<0.05)$.

\subsection{Altered ABA Sensitivity of Gs5PTase8-transgenic Arabidopsis}

Since 5PTase enzymes have been observed to be involved in ABA signaling pathway [7], ABA sensitivity were detected with the Arabidopsis plants over-expressing Gs5PTase8 in seed germination (Figure 6). The germination rates of all the plant lines declined when treated with the increased ABA for around $3.5 \mathrm{~d}$ after incubation at $21^{\circ} \mathrm{C}$. In general, the Gs5PTase8-transgenic lines showed less sensitive to ABA-elicited inhibition of seed germination compared to the wild type. With exposure to over $5 \mu \mathrm{M}$ ABA, all the seeds hardly germinated. To further verify the differences on ABA sensitivity between the transgenic lines and the wild type, we used $1 \mu \mathrm{M}$ ABA to perform the time course tests. It illustrated that the Gs5PTase8-transgenic plants germinated at a higher speed compared to the wild type. The ectopic expression of Gs5PTase8 could impart ABA insensitivity on seed germination, suggesting it might be involved in ABA signaling.

\subsection{Expression of the Stress-Responsive Genes in Gs5PTase8-Transgenic Arabidopsis}

To investigate whether the ectopic expression of Gs5PTase8 affects the stress-responsive genes, the expression levels of AtABA1, AtRD22, AtRD29A, and AtRD29B were detected in the Gs5PTase8-transgenic Arabidopsis lines and the wild type by qRT-PCR (Figure 7). AtABA1 participates in ABA synthesis, and the other three, AtRD22, AtRD29A, and AtRD29B have been showed to be induced by salt and other abiotic stresses [21,22]. All the tested genes were up-regulated in the first 3 or $6 \mathrm{~h}$ post salt treatment, then down-regulated and again up-regulated at $24 \mathrm{~h}$ post salt treatment. The expression of AtABA1 seemed not to be altered significantly by ectopic expression of Gs5PTase8 in Arabidopsis, indicating Gs5PTase 8 might not affect the ABA biosynthesis. On the other hand, the expression levels of the other three stress-responsive genes, AtRD22, AtRD29A, and AtRD29B, were induced more in the transgenic lines compared to the wild type under salt stress, suggesting Gs5PTase8 might take an important role in the regulation of those stress-responsive genes. 


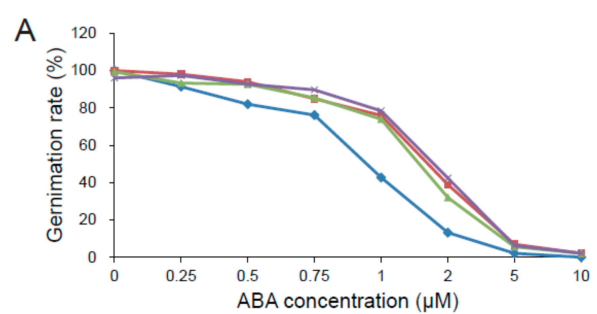

C

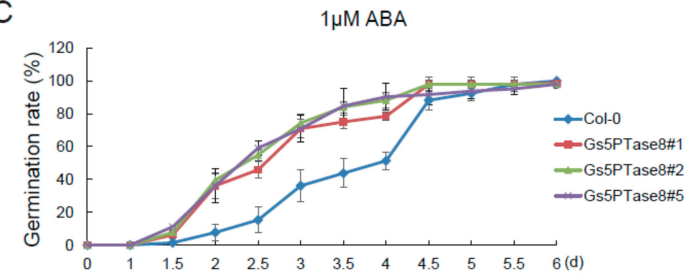

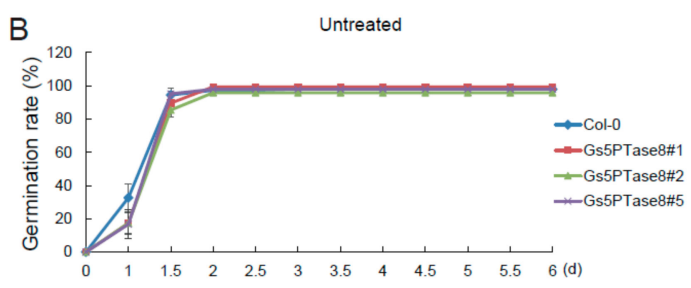

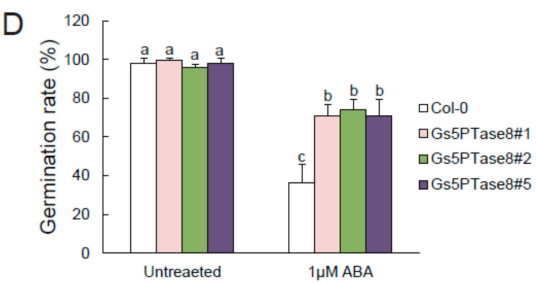

Figure 6. Germination of Gs5PTase8-transgenic Arabidopsis under abscisic acid (ABA) treatment. (A) The germination rate under a series of ABA treatment. Arabidopsis seeds were sowed on 1/2 MS median with varied $\mathrm{ABA}(0,0.25 \mu \mathrm{M}, 0.5 \mu \mathrm{M}, 0.75 \mu \mathrm{M}, 1 \mu \mathrm{M}, 2 \mu \mathrm{M}, 5 \mu \mathrm{M}$, and $10 \mu \mathrm{M})$, followed by being stratified at $4{ }^{\circ} \mathrm{C}$ for $3 \mathrm{~d}$ and then shifted to $21^{\circ} \mathrm{C}$. The germination rates were determined at around $3.5 \mathrm{~d}$ after seed imbibition for 150 seeds per line. (B) The germination rates were monitored every $12 \mathrm{~h}$ in 6 days after incubation at $21^{\circ} \mathrm{C}$ under normal conditions (untreated). (C) The germination rates under $1 \mu \mathrm{M}$ ABA treatment. (D) The statistical analysis of the germination rates at $3 \mathrm{~d}$ post stratification were performed by ANOVA with the Duncan's post hoc test $(p<0.05)$. The significant differences were indicated by different letters above the bars. A total of around 400-500 seeds were used for each sample from three independent experiments.

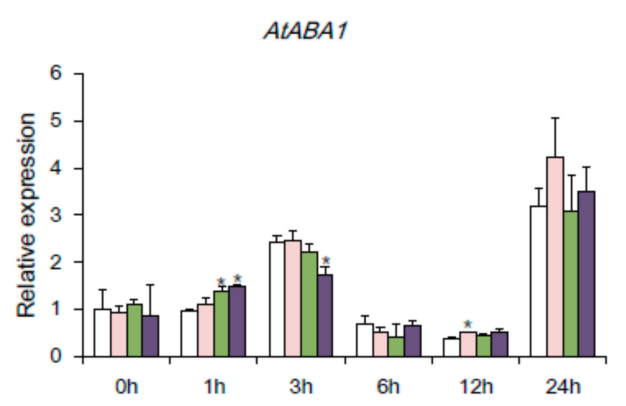

口Col-0 uGs5PTase8\#1 uGs5PTase8\#2 uGs5PTase8\#5

AtRD29A

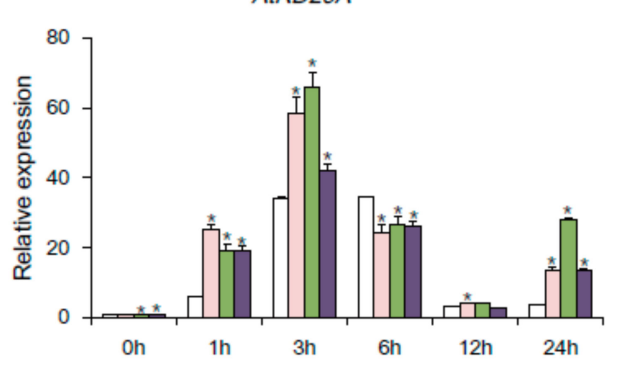

口Col-0 uGs5PTase8\#1 uGs5PTase8\#2 u Gs5PTase8\#5

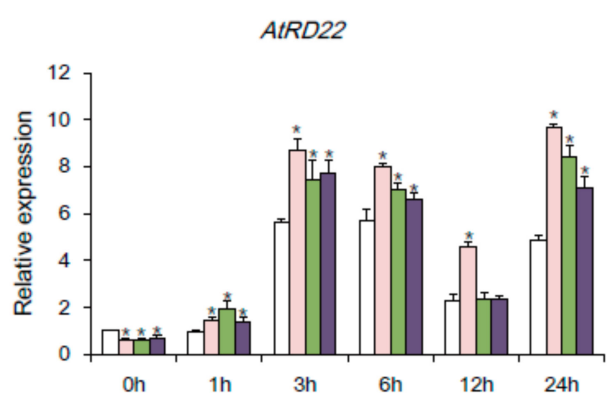

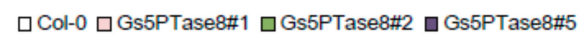

AtRD29B

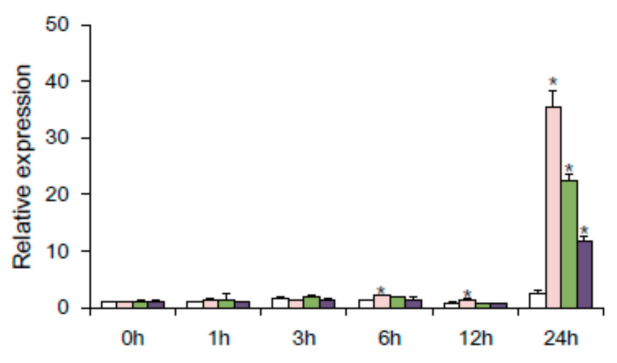

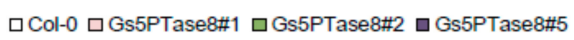

Figure 7. Effects of Gs5PTase8 on the expression levels of the stress-responsive genes. The three-week seedlings were treated by $100 \mathrm{mM} \mathrm{NaCl}$ in a hydroponic system for $0 \mathrm{~h}, 1 \mathrm{~h}, 3 \mathrm{~h}, 6 \mathrm{~h}, 12 \mathrm{~h}$, and $24 \mathrm{~h}$. The expression of AtABA1, AtRD22, AtRD29A, and AtRD29B were analyzed by qRT-PCR in the three independent Gs5PTase8-transgenic lines (Gs5PTase8 \#1, \#2, \#3) and the wild type (Col-0). The data were normalized to the reference gene ACT2. The value of the samples collected from Col- 0 at $0 \mathrm{~h}$ was set at 1 . The data were analyzed using ANOVA by the Dunnett's post hoc test. Asterisk indicates a significant difference between the wild type and the transgenic lines at the same time point $(p<0.05)$. 


\subsection{Subcellular Localization of Gs5PTase8}

To examine the subcellular localization of Gs5PTase8, the recombinant construct of Gs5PTase8-GFP under the control of the $35 \mathrm{~S}$ promoter were transformed into onion epidermal cells by particle bombardment (Figure 8). The 35S::GFP construct was used as the control. Before plasmolysis, the GFP signal appeared throughout the entire cell except the vacuole for 35S::GFP, whereas it was observed in the nucleus and plasma membrane for 35S::Gs5PTase8-GFP (Figure S1). After plasmolysis, the signals the Gs5PTase8-GFP protein were observed in the nuclear, plasma membrane, cell wall and especially in the apoplast (probably Hechitan strands), compared to the GFP alone protein. It revealed that Gs5PTase8 is expressed in the nucleus and plasma membrane. Besides, Gs5PTase8 targets to apoplast particularly, suggesting it might function in apoplastic pathways.

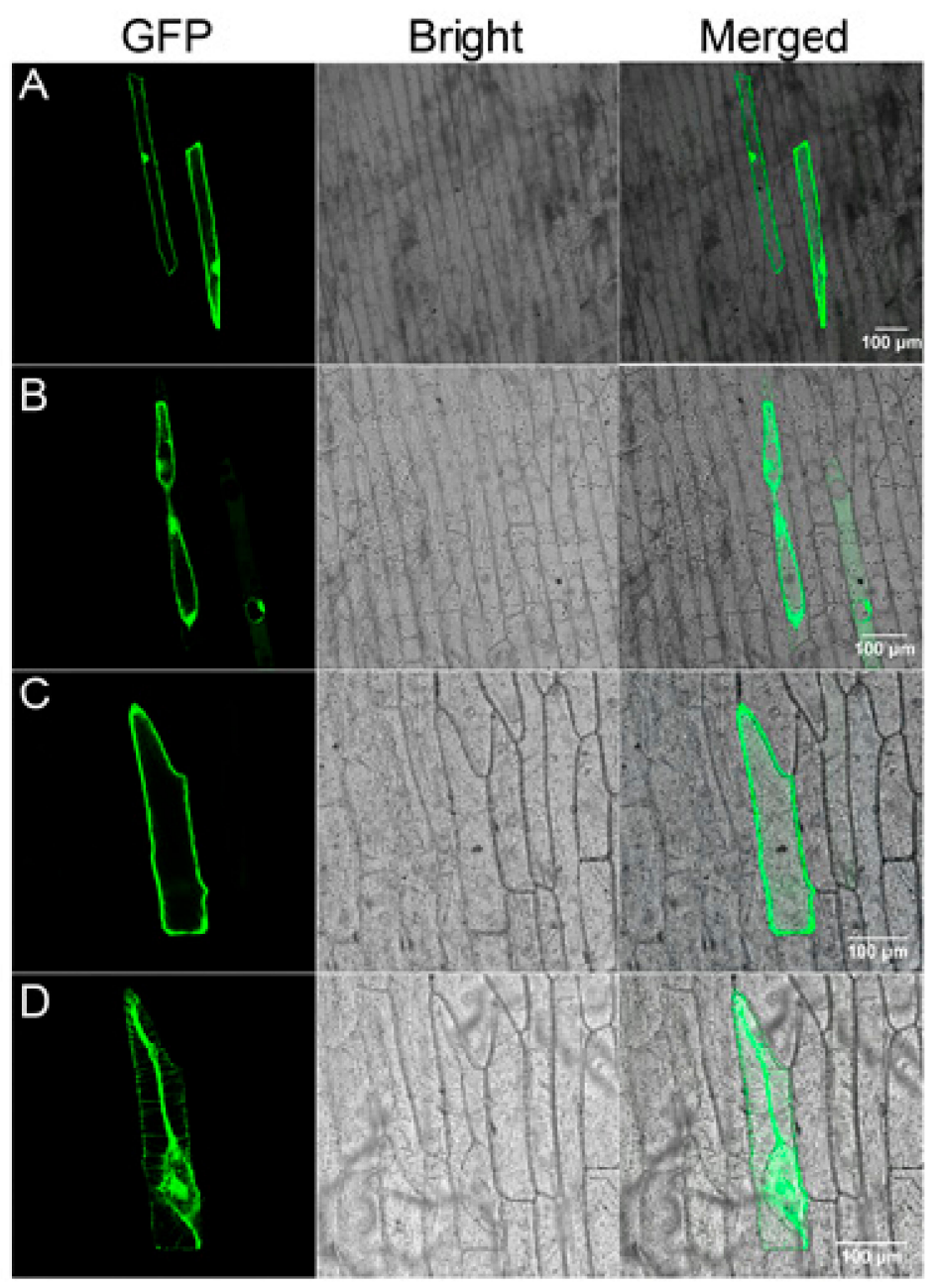

Figure 8. Subcellular localization of Gs5PTase8 in onion epidermal cells. Green fluorescence protein (GFP) (A, B) and Gs5PTase8-GFP (C, D) driven by CaMV 35S were transiently expressed in onion epidermal cells and observed before (A, C) or after (B, D) plasmolysis. GFP fluorescence, bright-field and merged images are shown. Scale bars indicate $100 \mu \mathrm{m}$.

\section{Discussion}

Inositol polyphosphate 5-phosphatases (5PTases) are one of the key components in inositol pathway $[8,9]$. Previous studies suggested that they could play an important roles in plant development and adaption to environmental stresses $[10,11]$. They have been identified to be widely distributed in all kingdoms of organisms [13]. However, only a few plant 5PTases have been investigated, and most are from the model plant, Arabidopsis thaliana. In this study, a novel Gs5PTase8 was cloned from Glycine soja 
and characterized for its function in salt responses. The sequence analysis illustrated that Gs5PTase8 contains the two conserved domain of the reported 5PTases (Figure 1), suggesting its potential function as an enzyme to hydrolyze the phosphate at the $5^{\prime}$ site of the inositol ring.

Based on the analyses of gene expression, Gs5PTase8 is highly induced in salt-tolerant accession W05. Moreover, the further functional analyses showed that ectopic expression of Gs5PTase8 could also confer salt tolerance in transgenic BY-2 cells (Figure 2), soybean hairy roots (Figure 3) and Arabidopsis (Figure 4). The activities of two antioxidant enzymes, CAT and APX, were increased in the Gs5PTase8-transgenic Arabidopsis lines under salt treatment (Figure 5), suggesting Gs5PTase8 might relieve the oxidative damage by salt stress and thus increase salt tolerance. All these revealed that Gs5PTase8 would be a positive regulator in salt tolerance, which might be a good candidate for soybean breeding.

5PTases have been found to affect ABA signaling pathway, which could play a vital role in plant adaption, especially to abiotic stresses [7,23]. We have determined the effect of Gs5PTase8 on ABA signaling. The results revealed that the overexpression of Gs5PTase8 in Arabidopsis caused ABA insensitivity on seed germination (Figure 6), which is similar as the previous reports on the mammalian type I 5PTase, At5PTase1, At5PTase2, and At5PTase6. The transgenic Arabidopsis plants expressing the mammalian type I 5PTase exhibited less sensitivity to ABA treatment and increased drought tolerance comparing to the wild type [24]. At5PTase1 and At5PTase2 could alter ABA signaling via the hydrolysis of inositol-1,4,5-trisphosphate ( $\mathrm{InsP}_{3}$ ) [25,26]. Their mutants showed a ABA hypersensitivity phenotype with faster seed germination and longer hypocotyls when grown in dark [27]. Similarly, the cop2 mutant (deficient in At5PTase6), with open vein network in leaf, also showed an increased sensitivity to ABA in seed germination [28]. But mutation in At5PTase13 caused ABA insensitivity [29,30]. Thus, it suggested that Gs5PTase8 might also impact ABA signaling for its function.

Among the reported Arabidopsis 5PTases, At5PTase7, and At5PTase9 have been characterized as a positive regulator in salt tolerance [14,15]. The overexpression of At5PTase7 or At5PTase9 displayed enhanced salt induction of the salt-induced marker genes, AtRD22 and AtRD29A [14,15]. We monitored the expression levels of three known stress-marker genes of $A t R D 22, A t R D 29 A$, and AtRD29B [31] under salt stress. The stress-marker genes could be up-regulated under abiotic stress or ABA treatment. Our observations showed that the expression of AtRD22, AtRD29A, and AtRD29B exhibited increased salt induction in Gs5PTase8-transgenic lines compared to the wild type as well (Figure 7). The subcellular localization analysis demonstrated that the fluorescent signal of Gs5PTase8-GFP could be detected in the nucleus. Gs5PTase8 might be involved in the regulation of stress-responsive gene expression by itself or via the ABA signaling pathway.

Except for the nuclear localization, Gs5PTase8-GFP was detected in plasma membrane with a relatively strong signal in the apoplast as well (Figure 8). The apoplast is the portion outside the cell membrane, including the cell wall and the intercellular space, with the function as a barrier and a linker between the environment and the protoplast [32]. Due to its apoplastic localization, we supposed that Gs5PTase8 might hydrolyze the membrane-bound phosphoinositide and be involved in the lipid signaling. However, there are no exploration on the substrates of Gs5PTase8 and its closest Arabidopsis homolog, At5PTase8, yet. Previous reports showed that various components exist in the apoplast, including ions, reactive oxygen species (ROS), and secreted proteins [32]. The apoplastic ROS and the scavenging enzymes have been identified to provide the signals for stress responses [33]. At5PTase7 and At5PTase9 have been identified to increase the production of ROS in the initial period of salt treatment, thus probably triggering the scavenging pathway to tolerate salt stress $[14,15]$. We have detected the activities of four antioxidant enzymes, CAT, APX, POD, and SOD at $24 \mathrm{~h}$ after salt treatment. Among them, CAT and APX were activated with a significant higher level in the Gs5PTase8-transgenic Arabidopsis plants than the wild type under salt treatment (Figure 5), suggesting that Gs5PTase8 would regulate ROS signaling probably in the apoplast under salt stress.

In conclusion, we identified a novel salt-induced Inositol polyphosphate 5-phosphatase gene, Gs5PTase8, in soybean and characterized its function in salt responses. The gain-of-function tests 
demonstrated that Gs5PTase8 conferred salt tolerance in transgenic BY-2 cells, soybean hairy roots and Arabidopsis. Overexpression of Gs5PTase8 increased the activity of CAT and APX, altered the ABA signaling pathway and up-regulated the expression of salt-responsive genes under salt stress, which might result in enhanced salt tolerance. Gs5PTase8 is localized not only inside the protoplast, but also the apoplast. This study provided a novel gene for improving salt tolerance in soybean and other plants. However, further investigation would be performed to reveal its substrates and its comprehensive roles in the ABA signaling and apoplastic pathways.

\section{Materials and Methods}

\subsection{Plant Materials and Growth Conditions}

The Glycine soja accession W05 (variety name: Mengjin1, salt tolerant) and Glycine max accession C08 (variety name: Union, salt sensitive) supplied by Dr. Honming Lam's lab, Chinese University of HongKong, were used for gene cloning and expression analyses in this work [17,34,35]. After soybean seeds were germinated on vermiculite for one week, the seedlings were transplanted to a hydroponic system of half-strength Hoagland's solution in a greenhouse [36].

Tobacco BY-2 cells [37] and Arabidopsis thaliana (ecotype: Columbia-0) were employed to obtain the transgenic lines for functional analyses. BY-2 cells were cultured in Murashige and Skoog (MS) media (Hopebio, Qingdao, China) supplemented with $0.025 \% \mathrm{KH}_{2} \mathrm{PO}_{4}$ and $3 \%$ sucrose at $25{ }^{\circ} \mathrm{C}$ in dark. Arabidopsis plants were grown on 1/2 MS medium or in hydroponic system of $1 / 10$ MS solution under a $16 \mathrm{~h}$ light $/ 8 \mathrm{~h}$ dark cycle at $21^{\circ} \mathrm{C}$ and $70 \%-80 \%$ humidity. The hydroponic system was used only for the gene expression analyses on Arabidopsis under salt treatment. And the plants were grown on $1 / 2$ MS medium for all the other experiments.

\subsection{Gene Expression Detected by $q R T-P C R$}

For soybean, when the first trifoliate of the soybean seedling opened, the nutrient solution was replaced by the fresh $1 / 2$ Hoagland's solution containing $0.9 \% \mathrm{NaCl}(w / v)[17,20,38]$. To extract the total RNA, the leaves and roots were collected and ground in liquid nitrogen separately at $0 \mathrm{~h}, 4 \mathrm{~h}, 24 \mathrm{~h}$, $48 \mathrm{~h}$, and $72 \mathrm{~h}$ after the start of salt treatments [20,38,39]. For Arabidopsis, three-week-old seedlings grown in the hydroponic system were transferred to the fresh 1/10 MS solution supplemented with $100 \mathrm{mM} \mathrm{NaCl}$. The sampling time points were $0 \mathrm{~h}, 1 \mathrm{~h}, 3 \mathrm{~h}, 6 \mathrm{~h}, 12 \mathrm{~h}$, and $24 \mathrm{~h}$ after the start of salt treatments. Three individual plants under the same treatment were pooled into one biological sample.

The total RNA was extracted using RNAiso PLUS reagent (TaKaRa Biotechnology Co. Ltd. Dalian, China) and the cDNA was synthesized using PrimeScript ${ }^{\mathrm{TM}}$ RT reagent Kit with gDNA Eraser (TAKARA, Dalian, China) according to the manufacturer's instructions. Quantitative reverse-transcription PCR (qRT-PCR) was performed using SYBR Premix Ex Taq ${ }^{\text {TM }}$ II (Tli RNaseH Plus) (TAKARA, Dalian, China) with the CFX96 Touch ${ }^{\text {TM }}$ Real-Time Detection System (Bio-Rad, Hercules, CA, USA). GmELF1b, $A t A C T 2$ and NtL25 were used as internal reference genes, respectively for soybean, Arabidopsis and tobacco BY-2 cells $[14,40,41]$. The gene specific primers are listed in Table S1. All the reactions were performed with three replicas. Relative gene expression was quantified using the $2^{-\Delta \Delta C t}$ method [42].

\subsection{Molecular Cloning and Sequence Analysis of Gs5PTase8}

First-strand cDNAs were synthesized with the RNA extracted from the soybean treated by salt stress, using a PrimeScript ${ }^{\mathrm{TM}}$ II 1st strand cDNA Synthesis Kit (TaKaRa, Dalian, China) following the manufacturer's protocol. Phusion High-Fidelity DNA polymerase (ThermoFisher Scientific, USA) was employed for amplifying the coding sequences of Gs5PTase8 with the gene specific primers (Table S1) [35]. The thermocycling conditions for PCR were as follows: $98{ }^{\circ} \mathrm{C} 30 \mathrm{~s} ; 35$ cycles of $98{ }^{\circ} \mathrm{C} 10 \mathrm{~s}, 58{ }^{\circ} \mathrm{C} 30 \mathrm{~s}$ and $72{ }^{\circ} \mathrm{C} 60 \mathrm{~s} ; 72{ }^{\circ} \mathrm{C} 10 \mathrm{~min}$ and $12{ }^{\circ} \mathrm{C}$ for hold. The amplicons with expected size were gel-purified and cloned to the pBluescript II KS (+) vector between the sites of $\mathrm{XbaI}$ and XhoI. The sequences of cDNAs were sequenced (BGI, Shenzhen) for verification. Then 
the cDNA was subcloned to the same restriction enzyme sites of the binary vector pTEV8 at the downstream of cauliflower mosaic virus $35 \mathrm{~S}$ promoter [43]. The recombinant construct was named as V7-Gs5PTase8. After sequence verification, they were transformed into Agrobacterium rhizogenes strain K599, Agrobacterium tumefaciens strain GV3101 or LBA4404 respectively by a freeze-thaw method [44].

The BLASTP searches were performed to find the homologous sequences (https://blast.ncbi.nlm. nih.gov/Blast.cgi). Multiple sequence alignment was performed with the full length sequences of protein using the CLUSTALX2.1 program [45]. The phylogenetic tree was constructed using MEGA version 6 by the neighbor-joining method with a bootstrap value of 1000 repeats [46].

\subsection{Cell Viability Assay of Transgenic BY-2 Cells}

Tobacco (Nitcotiana tabacum) BY-2 cells [37] were transformed with A. tumefaciens stain LBA4404 containing the recombinant plasmid, V7-Gs5PTase8 by a co-cultivation method [47]. The positive transformants were selected on the MS medium containing $50 \mathrm{mg} / \mathrm{L}$ kanamycin and the expression of the transgene was confirmed by qRT-PCR.

Four-day-old BY-2 cell suspension cultures were treated with $100 \mathrm{mM} \mathrm{NaCl}$ for $20 \mathrm{~h}$. The salt-treated and untreated cells were stained with $0.4 \%$ trypan blue (Sigma-Aldrich, St. Louis, MO, USA) for 5 min, and then were observed under a light microscope (Nikon Eclipse 80i, Tokyo, Japan). The images were captured by a CCD camera. A total of $200-300$ cells were counted for each sample. Three replicates were performed for each sample.

\subsection{Assays of Hairy Roots of Transformed Soybean Cotyledons under Salt Stress}

Hairy root transformation was performed as previously described with minor modifications [48]. The wild type of A. rhizogenes stain K599 (CK), K599 containing the empty vector (V), and K599 containing the recombinant construct V7-Gs5PTase8 (Gs5PTase8) were employed to transform cotyledons of any salt-sensitive soybean accessions (such as $\mathrm{C} 08$ and Williams 82) for hairy root induction. A. rhizogenes stains were grown in lysogeny broth (LB) medium containing $50 \mathrm{mg} / \mathrm{L}$ kanamycin and $200 \mu \mathrm{M}$ acetosyringone at $28^{\circ} \mathrm{C}$ for $16 \mathrm{~h}$. The soybean seeds were germinated with water at room temperature for 2-4 days. The cotyledons were cut off by a scalpel and scored by a syringe with needle. The wounds were infected with $A$. rhizogenes $\mathrm{K} 599$ for $15 \mathrm{~min}$ and then transferred to moist filter paper for co-cultivation in the dark at $25^{\circ} \mathrm{C}$ for 5 days. Subsequently, the infected cotyledons were transferred to a growth chamber under $12 \mathrm{~h}$ light $/ 12 \mathrm{~h}$ dark cycle at $25^{\circ} \mathrm{C}$. When the hairy roots were sprout, the infected cotyledons with the hairy roots of similar lengths (around $1 \mathrm{~cm}$ ) were selected and treated with $1 / 2$ Hoagland solution with or without $150 \mathrm{mM} \mathrm{NaCl}$ for 10 days. The hairy roots were photographed by a single lens reflex camera D7100 (Nikon Imaging (China) Sales Co., Shanghai, China) and the fresh weight of roots was measured. DNA was extracted respectively from each hairy root and the PCR reaction was performed with the NPTII primers (kanamycin selection marker of the binary vector pTEV8) to verify the positive transformation. The successful transformants of the empty vector and the recombinant construct would have a PCR product of $400 \mathrm{bps}$. The fresh weight data of those negative transformants were removed.

\subsection{Generation of Transgenic Arabidopsis Lines}

A. thaliana (ecotype: Col-0) plants were transformed by A. tumefaciens stain GV3101 containing the recombinant construct V7-Gs5PTase8 using the floral dip method [49]. The transgenic seeds were screened on MS medium with $50 \mathrm{mg} / \mathrm{L}$ kanamycin for positive transformants and at least 3 independent $\mathrm{T}_{1}$ lines were selected out. The homozygous $\mathrm{T}_{2}$ transgenic lines were obtained by selecting on MS medium with kanamycin and their progenies were used for further analysis. The expression of Gs5PTase8 was verified in the transgenic lines by qRT-PCR. 


\subsection{Phenotype Analyses of Transgenic Arabidopsis Lines}

For germination analyses, Arabidopsis seeds were sowed on normal 1/2 MS plates or the medium supplemented with $175 \mathrm{mM} \mathrm{NaCl}$ concentrations or varied $\mathrm{ABA}$ concentrations $(0.25 \mu \mathrm{M}, 0.5 \mu \mathrm{M}$, $0.75 \mu \mathrm{M}, 1 \mu \mathrm{M}, 2 \mu \mathrm{M}, 5 \mu \mathrm{M}$, or $10 \mu \mathrm{M})$ after surface-sterilization. The seeds were stratified at $4{ }^{\circ} \mathrm{C}$ in dark for $3 \mathrm{~d}$ before shifting to the growth chamber of $21^{\circ} \mathrm{C}$. Then, the germinated seeds were counted every $12 \mathrm{~h}$ in 6 days. To determine the best concentration for ABA sensitivity assay, around 150 seeds were used for each sample. In the other germination analyses, a total of around -500 seeds were used for each sample from three independent experiments.

For survival assays, the 3-day seedlings were transferred to the fresh 1/2 MS media with or without $175 \mathrm{mM} \mathrm{NaCl}$ for one week. A total of 75 plants were used for each sample from three replicates. The survival rate was calculated as the percentage of the survival plants after salt treatment. The chlorophyll content was extracted from $0.05 \mathrm{~g}$ of rosette leaves from the survival plants by being soaked in $1 \mathrm{~mL} \mathrm{95 \%} \mathrm{ethanol} \mathrm{at} 4{ }^{\circ} \mathrm{C}$ in dark for $24 \mathrm{~h}$. The absorbance of the extraction was checked at the wavelength of 649 and $665 \mathrm{~nm}$. Total chlorophyll concentration was calculated as the following equation: $\mathrm{C}_{\text {total }}=\mathrm{C}_{\mathrm{a}}+\mathrm{C}_{\mathrm{b}}=\left(\left(\mathrm{OD}_{665 \mathrm{~nm}} \times 13.95-\mathrm{OD}_{649 \mathrm{~nm}} \times 6.88\right)+\left(\mathrm{OD}_{649 \mathrm{~nm}} \times 24.96-\mathrm{OD}_{665 \mathrm{~nm}} \times\right.\right.$ 7.32))/(sample weight) [50].

\subsection{Assays for Antioxidant Enzyme Activities}

The $10 \mathrm{~d}$-seedlings were transferred to the $1 / 2 \mathrm{MS}$ media with or without $150 \mathrm{mM} \mathrm{NaCl}$ for $24 \mathrm{~h}$. Briefly, the fresh leaves of $0.1 \mathrm{~g}$ were ground in $2 \mathrm{~mL}$ of $0.2 \mathrm{M}$ phosphate buffer saline (PBS, $\mathrm{pH}$ 7.8) containing $1 \%$ polyvinylpyrrolidone (PVP) on ice. The homogenate was centrifuged at $4000 \mathrm{rpm}$ for half an hour at $4{ }^{\circ} \mathrm{C}$ and the supernatant was stored at $4{ }^{\circ} \mathrm{C}$ for the activity detection of catalase (CAT) [51], ascorbate peroxidase (APX) [52], superoxide dismutase (SOD) [53] and peroxidase (POD) [54]. The CAT activity was determined by monitoring decreases in absorbance at $240 \mathrm{~nm}$ due to the decomposition of $\mathrm{H}_{2} \mathrm{O}_{2}$. The APX activity was assessed by the decline of absorbance at $290 \mathrm{~nm}$ for the oxidation of ascorbate. The SOD activity was measured by detecting the absorbance at $560 \mathrm{~nm}$ for inhibiting the reduction of nitro blue tetrazolium (NBT) in light. The POD activity was assayed by detecting the absorbance at $470 \mathrm{~nm}$ due to the oxidation of guaiacol by $\mathrm{H}_{2} \mathrm{O}_{2}$. Each experiment was carried out at least three times.

\subsection{Subcellular Localization of Gs5PTase8}

The full length coding sequence of Gs5PTase8 without the stop codon was amplified and cloned into the $\mathrm{XbaI}$ and BamHI restriction enzyme sites of the binary vector PTEV8 at the downstream of 35S promoter. The GFP coding sequence was cloned behind Gs5PTase8 between the sites of BamHI and Xhol. The constructs were coated with gold particles and bombarded into the epidermal cells of onion (Bio-Rad PDS-1000/He system, Hercules, CA, USA). The onion sections were imaged at room temperature using a Leica SP8 X inverted confocal microscope with an Argon laser (Leica, Wetzlar, Germany). Green fluorescence protein (GFP) is excited at $488 \mathrm{~nm}$ and the emitted light is captured at 510-525nm. The images were captured digitally and processed using the Leica Application Suite Advanced Fluorescence Lite (LAS AF version: 2.6 .3 build 8173). Plasmolysis was performed by incubating the samples in $25 \%$ sucrose for $5 \mathrm{~min}$.

\subsection{Statistical Analyses}

Significant tests were performed by Statistical Package for Social Sciences (version 17.0, SPSS Inc. Chicago, IL, USA) statistical package.

Supplementary Materials: Supplementary materials can be found at http://www.mdpi.com/1422-0067/21/3/1023/s1.

Author Contributions: Conceptualization, Q.J., W.L. and J.H.; Data curation, Q.J., S.S., D.K. and L.W.; Funding acquisition, Q.J.; Investigation, Q.J., S.S., D.K., J.S., L.W., Z.Y., L.Z. and Y.Y.; Methodology, S.S. and L.W.; Project 
administration, K.L.; Resources, S.S., Z.Y. and K.L.; Supervision, Q.J., W.L. and J.H.; Writing-original draft, Q.J.; Writing - review and editing, Q.J. All authors have read and agreed to the published version of the manuscript.

Funding: This work was funded by National Natural Science Foundation of China (31501232), China Postdoctoral Science Foundation (2014T70603, 2013M540527), FAFU Science Fund for Distinguished Young Scholars (xjq201629), and open project of Key Laboratory of Ministry of Education for Genetics, Breeding and Multiple Utilization of Crops, Fujian Agriculture and Forestry University (GBMUC-2019-003).

Acknowledgments: We appreciate Honming Lam for kindly providing the BY-2 cells and soybean seeds of C08 and W05.

Conflicts of Interest: The authors declare no conflict of interest. The funders had no role in the design of the study; in the collection, analyses, or interpretation of data; in the writing of the manuscript, or in the decision to publish the results

\section{Abbreviations}

$\begin{array}{ll}\text { 5PTase } & \text { inositol polyphosphate 5-phosphatases } \\ \text { ABA } & \text { abscisic acid } \\ \text { APX } & \text { ascorbate peroxidase } \\ \text { CAT } & \text { catalase } \\ \text { InsP }_{3} & \text { inositol-1,4,5-trisphosphate } \\ \text { IPPC } & \text { inositol polyphosphate phosphatase catalytic } \\ \text { POD } & \text { peroxidase } \\ \text { ROS } & \text { reactive oxygen species } \\ \text { SOD } & \text { superoxide dismutase } \\ \text { WT } & \text { wild type }\end{array}$

\section{References}

1. Phang, T.-H.; Shao, G.; Lam, H.-M. Salt tolerance in soybean. J. Integr. Plant Biol. 2008, 50, $1196-1212$. [CrossRef] [PubMed]

2. Jha, U.C.; Bohra, A.; Jha, R.; Parida, S.K. Salinity stress response and 'omics' approaches for improving salinity stress tolerance in major grain legumes. Plant Cell Rep. 2019, 38, 255-277. [CrossRef]

3. Munns, R.; Tester, M. Mechanisms of salinity tolerance. Annu. Rev. Plant Biol. 2008, 59, 651-681. [CrossRef] [PubMed]

4. Hanin, M.; Ebel, C.; Ngom, M.; Laplaze, L.; Masmoudi, K. New Insights on Plant Salt Tolerance Mechanisms and Their Potential Use for Breeding. Front. Plant Sci. 2016, 7, 1787. [CrossRef] [PubMed]

5. Majerus, P.W.; Kisseleva, M.V.; Norris, F.A. The Role of Phosphatases in Inositol Signaling Reactions. J. Biol. Chem. 1999, 274, 10669-10672. [CrossRef] [PubMed]

6. Mitchell, C.A.; Gurung, R.; Kong, A.M.; Dyson, J.M.; Tan, A.; Ooms, L.M. Inositol Polyphosphate 5-Phosphatases: Lipid Phosphatases With Flair. IUBMB Life (International Union Biochem. Mol. Biol. Life) 2002, 53, 25-36. [CrossRef] [PubMed]

7. Jia, Q.; Kong, D.; Li, Q.; Sun, S.; Song, J.; Zhu, Y.; Liang, K.; Ke, Q.; Lin, W.; Huang, J. The Function of Inositol Phosphatases in Plant Tolerance to Abiotic Stress. Int. J. Mol. Sci. 2019, 20, 3999. [CrossRef]

8. Gillaspy, G.E. The cellular language of myo-inositol signaling. New Phytol. 2011, 192, 823-839. [CrossRef]

9. Xue, H.-W.; Chen, X.; Mei, Y. Function and regulation of phospholipid signalling in plants. Biochem. J. 2009, 421, 145-156. [CrossRef]

10. Hou, Q.; Ufer, G.; Bartels, D. Lipid signalling in plant responses to abiotic stress. Plant. Cell Environ. 2016, 39, 1029-1048. [CrossRef]

11. Heilmann, M.; Heilmann, I. Plant phosphoinositides—complex networks controlling growth and adaptation. Biochim. Biophys. Acta - Mol. Cell Biol. Lipids 2015, 1851, 759-769. [CrossRef] [PubMed]

12. Munnik, T.; Vermeer, J.E.M. Osmotic stress-induced phosphoinositide and inositol phosphate signalling in plants. Plant. Cell Environ. 2010, 33, 655-669. [CrossRef] [PubMed]

13. Zhang, Z.; Li, Y.; Luo, Z.; Kong, S.; Zhao, Y.; Zhang, C.; Zhang, W.; Yuan, H.; Cheng, L. Expansion and Functional Divergence of Inositol Polyphosphate 5-Phosphatases in Angiosperms. Genes (Basel). 2019, 10, 393. [CrossRef] [PubMed] 
14. Kaye, Y.; Golani, Y.; Singer, Y.; Leshem, Y.; Cohen, G.; Ercetin, M.; Gillaspy, G.; Levine, A. Inositol Polyphosphate 5-Phosphatase7 Regulates the Production of Reactive Oxygen Species and Salt Tolerance in Arabidopsis. Plant Physiol. 2011, 157, 229-241. [CrossRef]

15. Golani, Y.; Kaye, Y.; Gilhar, O.; Ercetin, M.; Gillaspy, G.; Levine, A. Inositol Polyphosphate Phosphatidylinositol 5-Phosphatase9 (At5PTase9) Controls Plant Salt Tolerance by Regulating Endocytosis. Mol. Plant 2013, 6, 1781-1794. [CrossRef]

16. Ku, Y.-S.; Koo, N.S.-C.; Li, F.W.-Y.; Li, M.-W.; Wang, H.; Tsai, S.-N.; Sun, F.; Lim, B.L.; Ko, W.-H.; Lam, H.-M. GmSAL1 hydrolyzes inositol-1,4,5-trisphosphate and regulates stomatal closure in detached leaves and ion compartmentalization in plant cells. PLoS ONE 2013, 8, e78181. [CrossRef]

17. Qi, X.; Li, M.-W.; Xie, M.; Liu, X.; Ni, M.; Shao, G.; Song, C.; Kay-Yuen Yim, A.; Tao, Y.; Wong, F.-L.; et al. Identification of a novel salt tolerance gene in wild soybean by whole-genome sequencing. Nat. Commun. 2014, 5, 4340. [CrossRef]

18. Guan, R.; Qu, Y.; Guo, Y.; Yu, L.; Liu, Y.; Jiang, J.; Chen, J.; Ren, Y.; Liu, G.; Tian, L.; et al. Salinity tolerance in soybean is modulated by natural variation in GmSALT3. Plant J. 2014, 80, 937-950. [CrossRef]

19. Patil, G.; Do, T.; Vuong, T.D.; Valliyodan, B.; Lee, J.-D.; Chaudhary, J.; Shannon, J.G.; Nguyen, H.T. Genomic-assisted haplotype analysis and the development of high-throughput SNP markers for salinity tolerance in soybean. Sci. Rep. 2016, 6, 19199. [CrossRef]

20. Liu, A.; Xiao, Z.; Li, M.-W.; Wong, F.-L.; Yung, W.-S.; Ku, Y.-S.; Wang, Q.; Wang, X.; Xie, M.; Yim, A.K.-Y.; et al. Transcriptomic reprogramming in soybean seedlings under salt stress. Plant. Cell Environ. 2019, 42, 98-114. [CrossRef]

21. Shinozaki, K.; Yamaguchi-Shinozaki, K. Gene networks involved in drought stress response and tolerance. J. Exp. Bot. 2007, 58, 221-227. [CrossRef] [PubMed]

22. Nambara, E.; Marion-Poll, A. Abscisic acid biosynthesis and catabolism. Annu. Rev. Plant Biol. 2005, 56, 165-185. [CrossRef]

23. Vishwakarma, K.; Upadhyay, N.; Kumar, N.; Yadav, G.; Singh, J.; Mishra, R.K.; Kumar, V.; Verma, R.; Upadhyay, R.G.; Pandey, M.; et al. Abscisic Acid Signaling and Abiotic Stress Tolerance in Plants: A Review on Current Knowledge and Future Prospects. Front. Plant Sci. 2017, 8, 161. [CrossRef]

24. Perera, I.Y.; Hung, C.-Y.; Moore, C.D.; Stevenson-Paulik, J.; Boss, W.F. Transgenic Arabidopsis Plants Expressing the Type 1 Inositol 5-Phosphatase Exhibit Increased Drought Tolerance and Altered Abscisic Acid Signaling. Plant Cell 2008, 20, 2876-2893. [CrossRef]

25. Sanchez, J.-P.; Chua, N.-H. Arabidopsis PLC1 Is Required for Secondary Responses to Abscisic Acid Signals. Plant Cell 2001, 13, 1143-1154. [CrossRef]

26. Burnette, R.N. An Arabidopsis Inositol 5-Phosphatase Gain-of-Function Alters Abscisic Acid Signaling. PLANT Physiol. 2003, 132, 1011-1019. [CrossRef]

27. Gunesekera, B.; Torabinejad, J.; Robinson, J.; Gillaspy, G.E. Inositol Polyphosphate 5-Phosphatases 1 and 2 Are Required for Regulating Seedling Growth. PLANT Physiol. 2007, 143, 1408-1417. [CrossRef]

28. Carland, F.M.; Nelson, T. COTYLEDON VASCULAR PATTERN2-Mediated Inositol $(1,4,5)$ Triphosphate Signal Transduction Is Essential for Closed Venation Patterns of Arabidopsis Foliar Organs. Plant Cell 2004, 16, 1263-1275. [CrossRef]

29. Ananieva, E.A.; Gillaspy, G.E.; Ely, A.; Burnette, R.N.; Erickson, F.L. Interaction of the WD40 Domain of a Myoinositol Polyphosphate 5-Phosphatase with SnRK1 Links Inositol, Sugar, and Stress Signaling. PLANT Physiol. 2008, 148, 1868-1882. [CrossRef]

30. Lin, W.-H.; Wang, Y.; Mueller-Roeber, B.; Brearley, C.A.; Xu, Z.-H.; Xue, H.-W. At5PTase13 Modulates Cotyledon Vein Development through Regulating Auxin Homeostasis. Plant Physiol. 2005, 139, 1677-1691. [CrossRef]

31. Zhu, J. Salt and Drought Stress Signal Transduction in Plants. Annu Rev Plant Biol 2002, 53, $247-273$. [CrossRef] [PubMed]

32. Zhang, L.; Tian, L.-H.; Zhao, J.-F.; Song, Y.; Zhang, C.-J.; Guo, Y. Identification of an apoplastic protein involved in the initial phase of salt stress response in rice root by two-dimensional electrophoresis. Plant Physiol. 2009, 149, 916-928. [CrossRef] [PubMed]

33. Mittler, R. Oxidative stress, antioxidants and stress tolerance. Trends Plant Sci. 2002, 7, 405-410. [CrossRef]

34. Xie, M.; Chung, C.Y.-L.; Li, M.-W.; Wong, F.-L.; Wang, X.; Liu, A.; Wang, Z.; Leung, A.K.-Y.; Wong, T.-H.; Tong, S.-W.; et al. A reference-grade wild soybean genome. Nat. Commun. 2019, 10, 1216. [CrossRef] 
35. Lam, H.-M.; Xu, X.; Liu, X.; Chen, W.; Yang, G.; Wong, F.-L.; Li, M.-W.; He, W.; Qin, N.; Wang, B.; et al. Resequencing of 31 wild and cultivated soybean genomes identifies patterns of genetic diversity and selection. Nat. Genet. 2010, 42, 1053-1059. [CrossRef]

36. Hoagland, D.R.; Arnon, D.I. The Water-Culture Method for Growing Plants Without Soil. Circ. Calif. Agric. Exp. Stn. 1950, 347, 1-32.

37. Nagata, T.; Nemoto, Y.; Hasezawa, S. Tobacco BY-2 Cell Line as the "HeLa" Cell in the Cell Biology of Higher Plants. Int. Rev. Cytol. 1992, 132, 1-30.

38. Jia, Q.; Xiao, Z.-X.; Wong, F.-L.; Sun, S.; Liang, K.-J.; Lam, H.-M. Genome-Wide Analyses of the Soybean F-Box Gene Family in Response to Salt Stress. Int. J. Mol. Sci. 2017, 18, 818. [CrossRef]

39. Bilgin, D.D.; DeLucia, E.H.; Clough, S.J. A robust plant RNA isolation method suitable for Affymetrix GeneChip analysis and quantitative real-time RT-PCR. Nat. Protoc. 2009, 4, 333-340. [CrossRef]

40. Yim, A.K.-Y.; Wong, J.W.-H.; Ku, Y.-S.; Qin, H.; Chan, T.-F.; Lam, H.-M. Using RNA-Seq Data to Evaluate Reference Genes Suitable for Gene Expression Studies in Soybean. PLoS ONE 2015, 10, e0136343. [CrossRef]

41. Schmidt, G.W.; Delaney, S.K. Stable internal reference genes for normalization of real-time RT-PCR in tobacco (Nicotiana tabacum) during development and abiotic stress. Mol. Genet. Genomics 2010, 283, $233-241$. [CrossRef]

42. Livak, K.J.; Schmittgen, T.D. Analysis of relative gene expression data using real-time quantitative PCR and the 2(-Delta Delta C(T)) Method. Methods 2001, 25, 402-408. [CrossRef] [PubMed]

43. Brears, T.; Liu, C.; Knight, T.J.; Coruzzi, G.M. Ectopic Overexpression of Asparagine Synthetase in Transgenic Tobacco. Plant Physiol. 1993, 103, 1285-1290. [CrossRef]

44. Weigel, D.; Glazebrook, J. Transformation of Agrobacterium Using the Freeze-Thaw Method. Cold Spring Harb. Protoc. 2006, 2006, pdb.prot4666. [CrossRef] [PubMed]

45. Larkin, M.A.; Blackshields, G.; Brown, N.P.; Chenna, R.; McGettigan, P.A.; McWilliam, H.; Valentin, F.; Wallace, I.M.; Wilm, A.; Lopez, R.; et al. Clustal W and Clustal X version 2.0. Bioinformatics 2007, 23, 2947-2948. [CrossRef] [PubMed]

46. Tamura, K.; Stecher, G.; Peterson, D.; Filipski, A.; Kumar, S. MEGA6: Molecular Evolutionary Genetics Analysis version 6.0. Mol. Biol. Evol. 2013, 30, 2725-2729. [CrossRef]

47. An, G. High Efficiency Transformation of Cultured Tobacco Cells. Plant Physiol. 1985, 79, 568-570. [CrossRef]

48. Wei, P.; Wang, L.; Liu, A.; Yu, B.; Lam, H.-M. GmCLC1 Confers Enhanced Salt Tolerance through Regulating Chloride Accumulation in Soybean. Front. Plant Sci. 2016, 7, 1082. [CrossRef]

49. Clough, S.J.; Bent, A.F. Floral dip: A simplified method for Agrobacterium-mediated transformation of Arabidopsis thaliana. Plant J. 1998, 16, 735-743. [CrossRef]

50. Butt, H.I.; Yang, Z.; Chen, E.; Zhao, G.; Gong, Q.; Yang, Z.; Zhang, X.; Li, F. Functional Characterization of Cotton GaMYB62L, a Novel R2R3 TF in Transgenic Arabidopsis. PLoS ONE 2017, 12, e0170578. [CrossRef]

51. Aebi, H. Catalase in vitro. Methods Enzymol. 1984, 105, 121-126. [PubMed]

52. Nakano, Y.; Asada, K. Hydrogen Peroxide is Scavenged by Ascorbate-specific Peroxidase in Spinach Chloroplasts. Plant Cell Physiol. 1981, 22, 867-880.

53. Giannopolitis, C.N.; Ries, S.K. Superoxide Dismutases. Plant Physiol. 1977, 59, 309-314. [CrossRef]

54. Maehly, A.C. The Assay of Catalases and Peroxidases. In Methods of Biochemical Analysis; John Wiley \& Sons, Ltd.: Hoboken, NJ, USA, 1954; pp. 357-424.

(C) 2020 by the authors. Licensee MDPI, Basel, Switzerland. This article is an open access article distributed under the terms and conditions of the Creative Commons Attribution (CC BY) license (http://creativecommons.org/licenses/by/4.0/). 